\title{
Fatigue as a risk factor for being injured in an occupational accident: results from the Maastricht Cohort Study
}

\author{
G M H Swaen, L G P M van Amelsvoort, U Bültmann, IJ Kant
}

Occup Environ Med 2003;60(Suppl I):i88-i92

See end of article for authors' affiliations

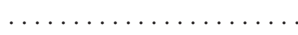

Correspondence to: Dr G Swaen, Department of Epidemiology, Maastricht University PO Box 616, 6200 MD Maastricht, Netherlands; g.swaen@epid.unimaas.nl

Accepted 12 September 2002

\begin{abstract}
Aims: To determine whether fatigue and need for recovery are risk factors for being injured in an occupational accident.

Methods: These associations were investigated within the Maastricht Cohort Study of "Fatigue at Work", a prospective cohort study of employees from a wide range of companies and organisations. For 7051 employees information was available on fatigue as measured with the Checklist Individual Strength (CIS), need for recovery as measured with the VBBA, and possible confounding factors such as age, smoking, alcohol consumption, educational level, shift work, and work environment. Information on the risk factors was collected in May 1999 and January 2000, before the occurrence of the occupational accidents. The incidence of being injured in an occupational accident was inventoried over the year 2000. A total of 108 employees reported having been injured in an occupational accident in 2000.

Results: For the highest CIS fatigue score tertile a for age, gender, educational level, smoking, shift work, and work environment, adjusted relative risk for being injured in an occupational accident of 1.29 (95\% Cl: 1.03 to 2.78 ) was found compared to the lowest tertile, and for the highest tertile of need for recovery a relative risk of $2.28(95 \% \mathrm{Cl}: 1.41$ to 3.66$)$ was found.

Conclusions: Fatigue and need for recovery were found to be independent risk factors for being injured in an occupational accident. This means that in the push back of occupational accidents, fatigue, and even more importantly need for recovery, need special attention.
\end{abstract}

( ccupational accidents are responsible for a significant proportion of worker absenteeism and disability. For the USA it has been estimated that occupational accidents cost approximately $\$ 145$ billion in 1992 compared to $\$ 26$ billion from all occupational illnesses combined. ${ }^{1}$ For the same year it was estimated that in the United States over 13 million occupational accidents occurred. The incidence of occupational accidents varies greatly from occupation to occupation and from industry sector to industry sector.

A number of demographic, lifestyle, and workplace factors have been reported to be associated with the risk of being injured in an occupational accident. These include age, ${ }^{2}$ alcohol consumption and smoking, ${ }^{3-6}$ shift work, ${ }^{78}$ and circumstantial factors - that is, factors describing the situational circumstances under which the accident took place. ${ }^{9}$ In their literature review, Hsiao and Simeonov present a model for structuring factors that play a role in falls from roofs, which can also be applied to occupational accidents in general. ${ }^{10}$ Their model consists of three categories of factors: work environment, task related factors, and personal factors. The work environment includes factors such as noise and machinery-in short, the physical environment in which tasks are performed. Task related factors describe the tasks of a certain job (for example, load handling, physical exertion, and complexity of the task). Personal factors include, for instance, age, training, and sensory interface with protective equipment. Fatigue is also often mentioned as a possible factor in the occurrence of accidents. The role of fatigue in the aetiology of accidents may be twofold: first, fatigue may decrease the ability to process information about a hazardous situation; and second, it may decrease the ability to adequately respond to a hazardous situation.

In their model Hsiao and Simeonov ${ }^{10}$ classify fatigue as a task related factor only, implying that performing tasks at a given intensity and duration can lead to fatigue. Fatigue may then decrease the ability of the worker to process important visual and perceptive information relevant to avoiding an accident. In contrast, fatigue can also be viewed as having a multifactorial origin, affected by non-work related circumstances and personal characteristics, with a prolonged character that may affect the individual's performance and ability to function at work. ${ }^{11}$ To date, it is unknown whether fatigue is a mediator between work related factors and the occurrence of accidents, or whether fatigue is an independent risk factor.

The aim of this study was to examine the longitudinal relation between fatigue and the incidence of being injured in an occupational accident. Two different aspects of fatigue are studied. These are "need for recovery" and prolonged fatigue. "Need for recovery" represents short term effects of a day of work and was defined as the need to recuperate from work induced fatigue primarily experienced after a day of work. ${ }^{12}$ The concept involves the intensity of work induced fatigue, both mentally as physically, as well as the time period required to return to a normal or prestressor level of functioning. In contrast, prolonged fatigue is seen as a subjective sensation, persistent over a period of several days, which affects the individual's performance in the work and home setting. ${ }^{14}$

Studies of risk factors for occupational accidents are hampered by a number of methodological constraints. One of these constraints is that most studies collect information on risk factors after the accident has occurred, which inevitably introduces recall bias. In a review of the epidemiology of occupational accidents, Burdorf et al stress the need for prospective cohort studies followed by targeted randomised experiments. ${ }^{15}$ The great advantage of prospective studies is that data on risk factors are collected before the accident occurred, thereby avoiding any recall bias as a result of the accident itself. The experience of being injured in an occupational accident can be so intense that the recollection of the factual situation in which the accident occurred will be distorted. Therefore we have chosen to investigate the effect of fatigue and need for recovery on the incidence of being injured in an occupational accident by means of a prospective study 
design. This investigation was based on data from the Maastricht Cohort Study on Fatigue at Work.

\section{MATERIALS AND METHODS}

\section{Study design}

The Maastricht Cohort Study of Fatigue at Work is a prospective study on a sample of 12140 workers from 45 different companies, mostly located in the southern part of the Netherlands. The design and rationale have been described elsewhere. ${ }^{16}{ }^{17}$

The cohort study started in May 1998 and covers a period of three years. In the cohort study information has been collected on a wide range of work related, health related, and demographic variables. For the analysis presented here, we used the data on demographic and work related variables that were collected in May 1999 as this was the closest point in time preceding the collection of the accident data for which these data were available. Information on fatigue and need for recovery was collected in January 2000, just before the period over which the incidence of injury in an occupational accident was monitored. Information on the incidence of being injured in an occupational accident was collected in the questionnaire that was mailed to the respondents in January 2001.

\section{Study population}

Of all participants at baseline (May 1998), 7051 employees filled in and returned the questionnaires mailed in May 1999, January 2000, and January 2001. All workers had at least a minimal employment of 50\% and were between 18 and 65 years of age. The study population formed a heterogeneous group of employees with different jobs and educational levels employed at one of 45 different companies that represented a range of different sectors and trades.

\section{Fatigue and need for recovery}

Fatigue was measured by means of the Checklist Individual Strength (CIS). This 20 item questionnaire was designed to measure several aspects of fatigue. It consists of four subscales: severity of fatigue, reduction in motivation, reduction in physical activity, and reduction in concentration. The CIS originates from the clinical setting and was constructed to test patients for chronic fatigue syndrome. The Cronbach's alpha has been reported to be $0.90{ }^{18}$ The CIS queries respondents about how they felt in the past two weeks. It consists of items such as: "I feel fit", "I am full of plans", "I have trouble concentrating", and "I feel like doing nice things". Respondents can answer on a seven point Likert scale. The 20 Likert scores are summed to give a CIS total ranging from 20 to 140. A pilot study conducted before to the start of the Maastricht Cohort Study indicated that a cut off point of $>76$ of the CIS has a sensitivity of $73 \%$ and a specificity of $90 \%$ to detect fatigued workers. ${ }^{17}$ We applied this a priori fixed cut off point to compare the risk for being injured in an occupational accident in fatigued workers with the risk in non-fatigued workers. Over time fatigue as measured with the CIS is a relatively stable factor. The CIS fatigue scores on the May 1999 and the January 2000 questionnaires had a correlation coefficient of 0.71 .

Need for recovery was measured with a subscale of the Dutch questionnaire on Perception and Judgement of Work. ${ }^{19}$ The scale consists of 11 items. Examples of the items included in this scale are: "I find it difficult to relax after a working day", "When I come home from work people should leave me alone", and "In my leisure time I have trouble concentrating". The total score has a range from 0 to 100 and has a Cronbach's alpha of 0.79. Because the respondent is asked to answer the questions with respect to a working day, it is thought that "need for recovery" measures acute effects of work on the respondent. Since there is no validated cut off point for this scale, we divided the population into tertiles.
Need for recovery must also be regarded as a relatively stable score. The correlation coefficient between the scores in May 1999 and January 2000 for the need for recovery scale was 0.76 .

\section{Confounders}

Possible confounding factors included demographic data (age, gender, and educational level), shift work, work environment, and lifestyle factors. Shift work has been reported to be an important risk factor for occupational accidents. ${ }^{7}$ As shift work is also related to fatigue, we identified shift work as a potential confounder in our investigation and included this factor in the analysis. This was done by using three categories: day shift only (between $7 \mathrm{am}$ and $7 \mathrm{pm}$ ), irregular shifts without night shift, and shift work with night shifts. The first category was used as the reference category.

As mentioned earlier, the type of work environment is an important class of risk factors for occupational accidents. We considered use of the variable industry sector to adjust for work environment. However, within a particular industry sector there can be more than one type of work environment and using sector as proxy measure for work environment would not catch the essential difference between, for instance, an office environment and an environment with machinery and tools. Therefore we recoded the jobs of all the study subjects into three classes of work environment. For each study subject, his/her job was coded according to the Dutch occupational classification designed by the Central Bureau of Statistics. ${ }^{20}$ Environment I consists of all subjects who perform office work or administrative work, with no exposure to machinery or tools except office machines. Environment II consists of all jobs that involve manual technical work and work with machinery and tools. Environment III consists of other jobs such as nursing, security, sales, and other jobs not characterised by office work (environment I) or manual work involving machines or tools (environment II). All the information mentioned above was collected in May 1999.

\section{Lifestyle factors}

Alcohol consumption was measured by asking about the weekly consumption in glasses of alcoholic beverages (drinking less than 14 glasses per week, or 14 or more). Smoking status was determined by the question: "Do you smoke every day?" (yes/no).

\section{Accidents at work}

In the literature there has been some debate about the use of the words "accident" and "injury" ${ }^{21}$ In that editorial the word "accident" was referred to as the " $\mathrm{A}$ " word, not to be used any longer, because of its overlap with injury or accident survival. In this study, the respondents were queried about being injured in an occupational accident, during the past year. The following item was used: "Did you have an accident at work in the previous year from which you suffered physical injury?" (response categories: yes/no). In addition we asked whether the subject had consulted a physician or physical therapist because of the suffered injury. Since we were interested in the serious accidents, we focused on those injuries for which the employee reported to have consulted a physician or physical therapist following the accident. Because of the debate in the literature about the use and misuse of the " $\mathrm{A}$ " word, we must be quite specific about the effect parameter used in our study. In this study the effect parameter refers to an employee who reported to have experienced an accident at work in which the worker suffered such physical injury that he consulted a medical doctor or physical therapist.

\section{Statistical analysis}

In a first step of the statistical analysis we investigated the univariate relations between the risk factors under study 
Table 1 Incidence of being injured in an occupational accident in different sectors

\begin{tabular}{ll}
\hline & $\begin{array}{l}\text { Crude incidence of being } \\
\text { injured in an } \\
\text { occupational accident } \\
\text { per } 100 \text { person-years of } \\
\text { observation }\end{array}$ \\
Type of industry sector (n) & $3.85^{*}$ \\
\hline Agriculture (26) & $1.90^{*}$ \\
Industry (3157) & $4.49^{*}$ \\
Public utilities (178) & 1.77 \\
Construction (113) & $2.11^{*}$ \\
Hotels/restaurants (95) & $3.63^{*}$ \\
Transportation and distribution (441) & 0.00 \\
Financial institutions (66) & 0.32 \\
Services (952) & 0.81 \\
Public administration (614) & 0.48 \\
Education (210) & 0.93 \\
Health/health care (1077) & 0.00 \\
Culture and recreation (122) & 1.53 \\
Total study population (7051) & \\
\hline *Indicates p<0.05, compared to the incidence of being injured in an \\
occupational accident in the services sector.
\end{tabular}

(fatigue and need for recovery) and the possible confounders with the risk of being injured in an occupational accident. Crude relative risks of the confounding factors for the incidence of being injured in an occupational accident and the corresponding $95 \%$ confidence intervals were calculated. Next, we calculated the crude relative risks for fatigue and need for recovery of being injured in a occupational accident. In a second step we adjusted for confounders that showed a confounding potency in the unadjusted analysis. Since fatigue as measured by the CIS and need for recovery are found to be correlated we additionally adjusted for need for recovery when fatigue was the independent variable under study and vice versa. All statistical analyses were performed by means of the SAS statistical computer program. Cox proportional hazard models were used to estimate the adjusted relative risks for being injured in an occupational accident.

\section{RESULTS}

In January 2001, 108 respondents of the Maastricht Cohort Study reported having been injured in an occupational accident, for which they consulted a physician or physical therapist.

The incidence of these accidents varied substantially between industry sectors. The highest incidence of being injured in an occupational accident was found in public utilities (4.5 per 100), agriculture (3.9 per 100), and transportation (3.6 per 100), whereas the lowest incidence was observed in the financial, educational, and cultural and recreational sectors (see table 1 ).

As has been reported by other investigators, the incidence of being injured in an occupational accident also varied with the age of the respondents. The lowest relative risk (RR) was found in the oldest age group, although the relative risks for the specific age groups did not significantly differ from unity (see table 2). Level of education was strongly associated with the risk for being injured in an occupational accident. Study subjects in the lowest educational group had approximately a sevenfold increased risk for being injured in an occupational accident compared to the group with the highest educational level (RR 7.38, 95\% CI: 3.64 to 14.98). The subgroup with a medium educational level had approximately a fivefold risk for being injured in an occupational accident compared to the study subjects with the highest educational level (RR 5.79, 95\% CI: 2.83 to 11.87 ).

Shift work was also strongly associated with the risk of being injured in an occupational accident. Shift workers with no night shifts had a nearly fivefold increased risk for being injured in an occupational accident compared to daytime employees (RR 4.76, 95\% CI: 2.42 to 9.35). Shift workers with night shifts had almost a threefold risk for being injured in an occupational accident compared to daytime employees (RR 2.74, $95 \%$ CI: 1.84 to 4.09 ).

Work environment was also strongly associated with the risk for being injured in an occupational accident. Workers with jobs in which they work with tools or machinery had a fivefold increased risk for being injured in an occupational accident (RR 5.03, 95\% CI: 3.32 to 7.63 ).

Smoking was also significantly related to the risk of being injured in an occupational accident (RR 2.04, 95\% CI: 1.38 to 3.00). Workers who reported they drank 14 glasses of alcoholic beverages or more per week did not significantly differ from the subjects who reported they drank less with respect to the risk of being injured in an occupational accident.

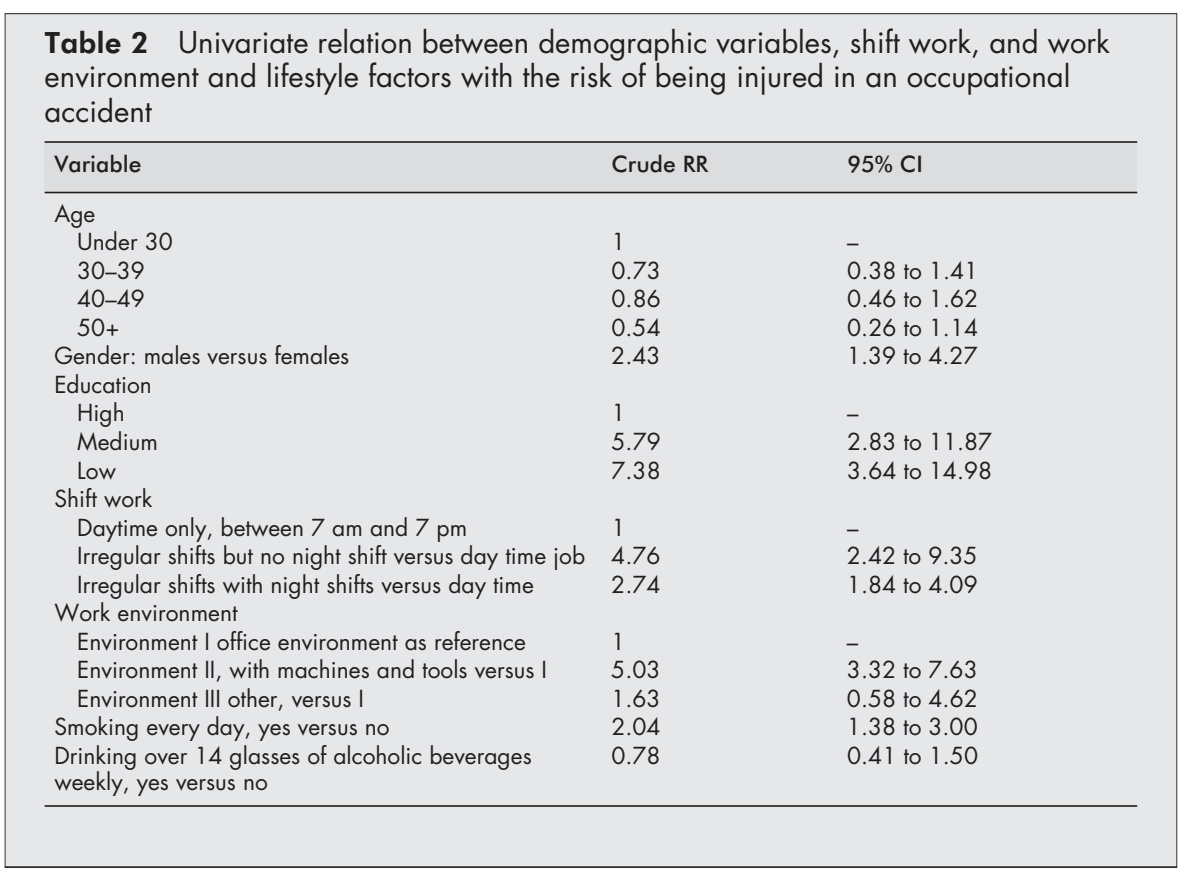




\begin{tabular}{|c|c|c|}
\hline Variable & Adjusted RR & $95 \% \mathrm{Cl}$ \\
\hline CIS: fatigued workers versus non-fatigued workers & 1.75 & 1.16 to 2.64 \\
\hline \multicolumn{3}{|l|}{ CIS total } \\
\hline Lowest tertile & 1 & - \\
\hline Medium tertile & 1.30 & 0.77 to 2.20 \\
\hline High tertile & 1.69 & 1.03 to 2.78 \\
\hline \multicolumn{3}{|l|}{ Subjective experience of fatigue } \\
\hline Lowest tertile & 1 & - $\quad$ n \\
\hline Medium tertile & 1.40 & 0.83 to 2.37 \\
\hline Highest tertile & 1.92 & 1.16 to 3.17 \\
\hline \multicolumn{3}{|l|}{ Reduced motivation } \\
\hline Lowest tertile & 1 & - \\
\hline Medium tertile & 1.30 & 0.79 to 2.13 \\
\hline Highest tertile & 1.47 & 0.91 to 2.38 \\
\hline \multicolumn{3}{|l|}{ Reduced activity } \\
\hline Lowest tertile & 1 & - \\
\hline Medium tertile & 0.93 & 0.56 to 1.54 \\
\hline Highest tertile & 1.19 & 0.74 to 1.91 \\
\hline \multicolumn{3}{|l|}{ Reduced concentration } \\
\hline Lowest tertile & 1 & - $\quad$ n \\
\hline Medium tertile & 0.69 & 0.42 to 1.15 \\
\hline Highest tertile & 1.16 & 0.74 to 1.80 \\
\hline \multicolumn{3}{|l|}{ Need for recovery } \\
\hline Lowest tertile & 1 & - \\
\hline Medium tertile & 1.41 & 0.80 to 2.47 \\
\hline Highest tertile & 2.28 & 1.41 to 3.66 \\
\hline
\end{tabular}

Both aspects of fatigue were found to be risk factors for being injured in an occpuational accident. Since adjustment for confounders (age, gender, education, smoking, shift work, and work environment) did not significantly change the unadjusted results we only presented the adjusted results (table 3). Employees scoring above the predefined cut off point for the CIS (fatigued workers) had a relative risk of 1.75 (95\% CI: 1.16 to 2.64) of being injured in an occupational accident compared to non-fatigued workers. The stratification of workers into three quartiles gave similar results; the highest tertile on the CIS score had a relative risk of 1.69 (95\% CI: 1.03 to 2.78) of being injured in an occupational accident compared to workers in the lowest tertile. Analysis of the subscales of the CIS revealed substantial differences. Where "subjective experience of fatigue" was significantly associated with the risk of being injured in an occupational accident, the other three dimensions of the CIS were only marginally associated (table 3).

Need for recovery also appeared to be related to the risk of being injured in an occupational accident. Workers in the highest tertile of the need for recovery scale had a relative risk of 2.28 (95\% CI: 1.41 to 3.66 ) for being injured in an occupational accident.

The relation between fatigue caseness and the risk of being injured in an occupational accident decreased and became not statistically significant (RR 1.31, 95\% CI: 0.75 to 2.27 ) after additional adjustment for need for recovery. The relative risk for need for recovery and the risk of being injured in an occupational accident, however, was not affected by additional adjustment for fatigue. In fact the relative risk for the highest tertile increased from 2.28 to 2.60 .

\section{DISCUSSION}

This study provides evidence that both fatigue and need for recovery are independent risk factors for being injured in an occupational accident. The results are based on 108 workers who reported to have been injured in an occupational accident in a period of one year in a heterogeneous group of employees from 45 different companies. For fatigue, the adjusted relative risk for being injured in an occupational accident was 1.69
(95\% CI: 1.03 to 2.78 ) when comparing workers scoring in the highest tertile on the CIS, with workers scoring in the lowest tertile. For need for recovery, the adjusted relative risk for workers scoring in the highest tertile, compared to workers scoring in the lowest tertile was 2.28 (95\% CI: 1.41 to 3.66). For both measures a dose-response relation was found. Surprisingly the effects on the incidence of occupational accidents occurred already on low to moderate levels of fatigue and need for recovery.

The univariate analysis also revealed a strong relation between age, gender, educational level, smoking, shift work, and work environment and the risk of being injured in an occupational accident. However, additional adjustment of the relation between fatigue and need for recovery and the risk of being injured in an occupational accident for these factors revealed only minor differences. This strengthens the hypothesis that fatigue and need for recovery can be regarded as independent risk factors.

An earlier study within the Maastricht Cohort Study revealed that need for recovery as measured with the VBBA is associated with prolonged fatigue as measured with the CIS (Pearson correlation coefficient 0.63 ). This suggests a possible overlap between these two constructs. However, in the same study, a principal component analysis revealed obvious separation between need for recovery items and CIS items, supporting the notion that need for recovery and fatigue represent different underlying concepts. In this study, the relation between fatigue and the risk of being injured was additionally adjusted for need for recovery and vice versa. When additionally adjusted for need for recovery, the relative risk of fatigue decreased and was no longer statistically significant. However, additional adjustment of need for recovery for fatigue increased the relative risk of need for recovery on being injured in an occupational accident. As we regard fatigue and need for recovery as different constructs, it can be concluded that need for recovery dominates the risk of being injured in an occupational accident.

Several methodological issues have to be considered. First, the study design allowed that data on independent variables were collected before an occupational accident occurred. Thus, 
it is impossible that the scores on the risk factors have been influenced by the fact that a study subject had been injured in an occupational accident. Therefore we consider it unlikely that the study results are affected by information bias. However, it cannot be totally ruled out that the fatigue level reported in January 2000 may have had some effect on reporting an injury in an occupational accident in January 2001.

Second, data on being injured in an occupational accident in this study were based on self report and could not be verified by an independent observer. This might be a source of information bias. However, the incidence data and the associations with age, smoking, education, shift work, and work environment are all in line with the literature.

Third, it is important to keep the timeframe of the study in mind. One issue concerns the time span between the assessment of demographic, lifestyle, and work related factors, which reflect the condition of May 1999 and the reporting of being injured in an occupational accident over 2000. However, we regarded demographic, lifestyle, and work related factors as relatively stable factors. Also the data on fatigue and need for recovery reported in January 2000 have been assumed to be representative for the state of fatigue and need for recovery at the time of the injury in an occupational accident. However, fatigue or need for recovery may have changed over the year 2000. The correlation coefficients for fatigue and need for recovery between May 1999 and January 2000 are over 0.7 , indicating that this misclassification has been small. As these changes are probably not related to the risk for being injured in an occupational accident, the bias is likely to be towards the null.

To conclude, in this prospective cohort study, we found significant relative risks for being injured in an occupational accident for both for fatigue as well as for need for recovery. This means that in the push back of occupational accidents, fatigue, and even more importantly need for recovery, need special attention.

\section{ACKNOWLEDGEMENT}

This study is part of the Netherlands concerted research action on "Fatigue at Work" granted by the Netherlands Organisation for Scientific Research (NWO) under grant number 580-02.254.

\section{Authors' affiliations}

G M H Swaen, L G P M van Amelsvoort, U Bültmann, IJ Kant, Department of Epidemiology, Maastricht University, Maastricht, Netherlands

\section{REFERENCES}

1 McDonald C. Epidemiology of work related diseases. London: BM Books, 2000.

2 Laflamme L, Menckel E, Lundholm L. The age-related risk of occupational accidents: the case of Swedish iron-ore miners. Accid Analy Prev 1996;28:349-57.

3 Chipman ML. Risk factors for injury: similarities and differences for traffic crashes and other causes. Accid Analy Prev 1995;27:699-706.

4 Frone MR. Predictors of work injuries among employed adolescents. J Appl Psychol 1998;83:565-76.

5 Wells S, Macdonald S. The relationship between alcohol consumption patterns and car, work, sports and home accidents for different age groups. Accid Analy Prev 1999;31:663-5.

6 Leistikow BN, Martin DC, Jacobs J, et al. Smoking as a risk factor for accident death: a meta-analysis of cohort studies. Accid Analy Prev 2000;32:397-405

7 Frank AL. Injuries related to shiftwork. Am J Prev Med 2000;18|4 suppl):33-6.

8 Nag PK, Patel VG. Work accidents among shift workers in industry. International Journal of Industrial Ergonomics 1998;21:275-81.

9 Veazie MA, Landen DD, Bender TR, Amandus HE. Epidemiologic research on the etiology of injuries at work. Annu Rev Public Health 1994; 15:203-21.

10 Hsiao H, Simeonov P. Preventing falls from roofs: a critical review. Ergonomics 2001;44:537-61.

11 Lewis G, Wessely S. The epidemiology of fatigue: more questions than answers. J Epidemiol Community Health 1992;46:92-7.

12 Sluiter JK, Frings-Dresen MH, van $\operatorname{der}$ Beek AJ, et al. The relation between work-induced neuroendocrine reactivity and recovery, subjective need for recovery, and health status. J Psychosom Res 2001;50:29-37.

13 Meijman T. Mentale belasting en werkstress. Een arbeidspsychologische benadering [Mental strain and workstress. An I/O psychology approach]. Assen/Maastricht: Van Gorcum, 1989

14 Meijman T, Schaufeli W. Psychische vermoeidheid en arbeid. Ontwikkelingen in de A\&O-psychologie [Fatigue at work. Developments in I\&O psychology]. De Psycholoog 1996;June:236-41.

15 Burdorf A, Sorock GS, Herrick RF, et al. Advancing epidemiologic studies of occupational injury-approaches and future directions. Am J Ind Med 1997;32:180-3.

16 Kant IJ, Bültmann U, Schröer KAP, et al. An epidemiological approach to study fatigue in the working population: the Maastricht Cohort Study. Occup Environ Med 2003;60(suppl 1):i32-9.

17 Bültmann U, De Vries M, Beurskens AJHM, et al. Measurement of prolonged fatigue in the working population: determination of a cutoff point for the Checklist Individual Strength. J Occup Health Psychol 2000;5:411-16.

18 Vercoulen JH, Swanink CM, Zitman FG, et al. Randomised, double-blind, placebo-controlled study of fluoxetine in chronic fatigue syndrome. Lancet 1996;347:858-61.

19 Van Veldhoven M, Meijman T. Het meten van psychosociale arbeidsbelasting met een vragenlijst: De Vragenlijst Beleving en Beoordeling van de Arbeid (VBBA) [Questionnaire on Perception and Judgement of Work]. Amsterdam: NIA, 1994.

20 CBS. Standaard beroepenclassificatie [Dutch classification of occupations]. 's Gravenhage, The Netherlands: Staatsuitgeverii, 1993.

21 Davis RM, Pless B. BM bans "accidents". BM 2001;322:1320-1. 